\title{
Health Resources Allocation Analyzing of the Western China
}

\section{Zheng Wang}

Guangxi Medical University

\section{Haoyu He}

Guangxi Medical University college of stomatology

Xi Liu

Guangxi Medical College

Qiming Feng

Guangxi Medical University

Bo Wei (D 583400698@qq.com )

Guangxi Medical University

\section{Research Article}

Keywords: Western China, Health resources allocation, Gini coefficient

Posted Date: December 15th, 2020

DOI: https://doi.org/10.21203/rs.3.rs-122801/v1

License: (c) (1) This work is licensed under a Creative Commons Attribution 4.0 International License. Read Full License 


\section{Health resources allocation analyzing of the western}

China

Zheng Wang1,3, Haoyu He², Xi Liu1,3, Qiming Feng 1, Bo Wei 1

'School of Information and Management, Guangxi Medical University, Shuangyong Road, Nanning, Guangxi, 530021, China.

${ }^{2}$ Quality Management Department, the Affiliated Hospital of Stomatology, Guangxi Medical University, 10 Shuangyong Road, Nanning, 530021, China

${ }^{3}$ Guangxi Medical College, 8 Kunlun Road, Nanning, Guangxi,530000, China

\section{Corresponding author}

Correspondence to Bo Wei, email: 583400698@qq.com

\section{Abstract}

Background: To achieve the goal "health equity for all", equitable and reasonable allocation of health resources is the basic material guarantee. As an area which owns more developing provinces and minority nationality region than other regions in China, the western China should get more attention in the health resources allocation while there was not. 
Methods: Lorentz curve, Gini coefficient and Theil index were used to analyze the health resources allocation which including number of health institutions, number of beds, (assistant) medical practitioners, registered nurses in the western China from 2014 to 2018 from two dimension: population and geography.

Results: The total health resources shows an increasing tendency from 2014-2018; The Gini coefficients for health resources by population dimension were ranged from 0.057 to 0.13 , in geography dimension the Gini coefficients ranged between 0.61 and 0.64 . Meanwhile, the Lorentz curve in population dimension had a smaller curvature than in geography dimension; In two dimensions, the intra-group contribution rate of the Theil index was higher than the inter-group.

Conclusion: The equity of health resources allocation should be further improved, especially the allocation of health institutions. Moreover, the access to health resources should also by improve by a diversity method.

\section{Key Word}

Western China; Health resources allocation; Gini coefficient

\section{Introduction}

The "Healthy China 2030" Plan which released in 2016 was clearly 
emphasized that "Gradually reduce the differences in basic health services and health levels between urban and rural areas, regions and populations to achieve universal health coverage and promote social equity" [1].To achieve this goal, what is the most significant is optimizing the allocation of health resources and ensure the fair distribution of resources [2].According to the previous research, as a developing region, the equity of the allocation of health resources in the western China was lower than that in other developed regions [3], which requires more attention but still as shortage. Hence, a research which focus on the equity of the allocation of health resources in the western China seems particularly necessary, and it had also become one of a research hot pot in the recent years.

Base on the background above, in this paper, by using the Gini coefficient, Lorentz curve and Theil index, we analyzed the equity of health resource allocation in the western China, to further promote the equity of health resource allocation, some suggestions were put forward according to the analysis results at the end.

\section{Data sources Methods}

\section{Functional regional division}

According to NDRC, the western of China includes 12 provinces, namely

Shaanxi, Sichuan, Yunnan, Guizhou, Gansu, Qinghai, Guangxi Zhuang 
Autonomous Region, Ningxia Hui Autonomous Region, Tibet Autonomous Region, Xinjiang Uygur Autonomous Region, Inner Mongolia Autonomous Region and Chongqing Municipality. Ethnic minority autonomous regions include The Guangxi Zhuang Autonomous Region, in this paper, we divided the 12 provinces into two area: Ethnic minority area Non-ethnic minority areas. Ethnic minority area includes the Ningxia Hui Autonomous Region, the Tibet Autonomous Region, the Xinjiang Uygur Autonomous Region and the Inner Mongolia Autonomous Region, the rest of the provinces were divided into nonminority areas.

\section{Data sources and statistical analysis}

The data in this paper were mainly searched from China statistical yearbook from 2015 to 2019, and the public data which released by the National Health and Family Planning Commission from 2015 to 2019. During searching data what we mainly focused on was the number of beds, heath institutions, (assistant) medical practitioners, registered nurses in the western of China from 2014-2018. After searching data, we established a database by using excel2010 to further process data.

\section{Lorentz curve}

Lorenz curve is one of the mainly tools to evaluate the equity of resources 
or income distribution by income or resources classified according to different groups or regions, after that, coordinate system is constructed with the accumulated percentage of population or land area and the accumulated percentage of various medical resources. Then, draw the coordinate point connected to the zero point and each curve. The fairness is judged according to its distance from the absolute fairness line. The closer it is to the absolute fairness line, the fairer it is, and the worse it is[4].

\section{Gini coefficient}

Lorentz curve can evaluate the equity of resources. However, it cannot quantify the specific differences of equity[5], hence, the Gini coefficient is often used to fill this gap. The Gini coefficient is calculated as follows:

$$
G=\frac{1}{2 n^{2} \mu} \sum_{i=1}^{n} \sum_{j-1}^{n}\left|y_{i}-y_{j}\right|
$$

Where, $\mathrm{G}$ stands for Gini coefficient, $\mathrm{N}$ for group number, $\mu$ for resident income, $Y_{i}$ and $Y_{j}$ for per capita income of group I and J[6].

According to the researches by Zhang nan, Peng rong et al, The Gini coefficient value range is $[0,1]$.The closer it gets to 0 , the fairer it is. On the other hand, the closer it is to 1 , the less fair it is. It is generally believed that: Gini coefficient less than 0.3 means the resources allocation is in a best fair state;0.3 0.4 is the normal state; $A$ level of alert above 0.4 ; Above 
0.6 is in a dangerous state of high unfairness $[7,8]$.

\section{Theil index}

Theil index is the major tool to measure fairness, and its value range is $[0,1]$. The smaller the value is, the better its fairness is. Its calculation formula is:

$$
\begin{array}{ll}
\text { Theil }-l=\sum_{i-1}^{n} P_{n} \log _{\overline{P_{n}}} & \text { (formula 1) } \\
T_{\text {inter }}=\sum_{g-1}^{k} P_{j} \log _{\overline{P_{j}}} & \text { (formula 2) } \\
T_{\text {intra }}=\sum_{g-1}^{k} P_{j} T_{j} & \text { (formula 3) } \\
T \text { heil }=T_{\text {inter }}+T_{\text {intra }} & \text { (formula 4) }
\end{array}
$$

Where, $P_{n}$ refers to the proportion of the population of each province in the total population; $P_{j}$ is the proportion of the population of each region in the total population; Each $y_{n}$ is the proportion of resources of each province in the total number of resources on this dimension. $y_{j}$ shows the proportion of resources of each region in the total number of resources $[9,10]$

Results

The basic situation of health resources allocation in the western China from 2014 to 2018

According to table 1 to Table 3, the distribution of health resources in 12 
provinces in western China from 2014 to 2018 showed a trend of continuous growth. Among them, Tibet had the worst health resource allocation overall. By 2018, Tibet had 0.5 health institutions, 4.88 beds, 2.44 occupational (assistant physician) and 1.62 registered nurses per 1,000 people. In terms of the distribution of health institutions, Yunnan had the highest level which attained 1.55 health institutions per 1,000 people in 2018; In the aspect of beds distribution, Xinjiang were than other provinces in the western China with 7.19 beds per 1,000 people in 2018. As for the distribution of health technicians, Inner Mongolia was the best among other provinces in the western China with 2.90 licensed (assistant) physicians and 3.02 registered nurses per 1,000 people in 2018

\section{Lorentz curve of health resources in western China from 2014 to 2018}

From the perspective of Lorentz curve analysis, Figure 1 shows the result that the Lorentz curve based on population dimension was closer to the absolute fairness line overall than based on geographical dimension. Among them, based on population dimension, health institutions got a more curved Lorentz curve than others. Furthermore, we can see from Figure 2 that the degreed of bending of the four curves were similar based on geographical dimension, compared with the Lorentz curve in the population dimension (Fig. 1), they were further away from the absolute fairness line. This indicates that the distribution of health 
resources in geographical dimension had less equitable than that according to population dimension.

Gini coefficient of health resources in western China from 2014 to 2018

According to the Gini coefficient analysis in western China from different dimensions (Table 4), the Gini coefficients of beds, health institutions, practicing (assistant) physicians and registered nurses calculated in geographical dimension were between 0.60 and 0.63 , showing a highly unfair state, while the four health resources were in the best fair state with all Gini coefficients were less than 0.2 in population dimension. Meanwhile, in the population dimension, the distribution of beds was best the other three, with the Gini coefficient between 0.057 and 0.058 .

In addition, base on the Figure 3, we can see that the Gini coefficient in population dimension present a downward trend, meanwhile, the Gini coefficient of registered nurses showed the most obvious downward trend in 2017 among the four health resources, while the Gini coefficient based on geographical area presents an overall upward trend which can be seen in the Figure 4.

According to the results above we can draw a conclusion that the distribution of beds based on population dimension was most equitable, 
the improvement effect of the equity of registered nurses was the most obvious, and the inequality of the distribution of health resources based on geographical dimension tends to worsen.

\section{Theil index of health resources in western China}

Base on the Thiel index analysis of different dimensions that shown in Table 5 and 6, the total Thiel index of health resources in the two dimensions presented tendency of $T_{\text {population }}>T_{\text {geographical. }}$ Moreover, the Theil index in the two dimensions shows a status of intra-group higher than inter-group. This result implied that the distribution of health resources was more equitable in the population dimension than in the geographical dimension, and the inter-group also had a higher equity of health resources allocation than the inter group.

\section{Thiel index contribution rate analysis}

From the perspective of population dimension, beds, health institutions, medical (assistant) practitioners and nurses were all showed that the intra-group contribution rate greater than the inter-group contribution rate. Among them, the contribution rate of both health institutions and registered nurses in the group reached more than $90 \%$ (Table 7). Meanwhile, contribution rate of licensed (assistant) physicians was kept rise, and the contribution rate of beds present a decline tendency. The 
above results indicated that the inequity of health resource allocation based on population and geographical dimension was mainly came from intra-groups (Table 8). In the population dimension, and health institutions and registered nurses existed the most obvious intra-groups inequity distribution. Moreover, the intra-groups inequity distribution of medical (assistant) practitioners showed an increase tendency, while it was decreased in beds distribution.

\section{Discussion}

The overall health resources showed an increasing trend, while the equity improvement was not obvious except for registered nurses

From 2014 to 2018, per one thousand people have health institutions, health and technical personnel and the number of beds, registered nurses in western China showed a trend of steady growth which indicated that Chinese government got a satisfactory result in increasing health resources, and residents' medical needs had been better meet. Among them, nurses per 1,000 had the most significant increase, Yunnan and Chongqing had an average annual growth rate of $23 \%$. In comparison, the growth rate of health institutions per thousand people was relatively slow, the most significant growth rate was in Xinjiang with only $2.3 \%$. Moreover, Ningxia, Chongqing and Inner Mongolia had a negative growth rate with $-0.05 \%$, indicated that the allocation of health 
institutions need to be strengthened, which is consistent with the research results of Ma Lu et al. [11]

Although health resources in western China present a growing trend but expect for the register nurses got a relative obvious improvement effect with Gini coefficient changed from 0.071 to 0.041 in the population dimension, other health resources allocation equity did not have an obvious improvement. In addition, the number of health institutions Gini coefficient is relatively high which means its fairness is poorer among the four health resources.

The geographical dimension of health resource allocation equity needs to be improved

According to the Gini coefficient analysis in different dimensions, the Gini coefficients of the number of health institutions, beds, medical (assistant)practitioners and registered nurses in the geographic dimension were higher than those in the population dimension, with the coefficients that all higher than 0.6 which present a highly unfair state, and it also implied that health resources allocation fairness has great improvement in geographical dimension. This can be owing to that currently the main way China choose to allocation the health resources was based on population. Meanwhile, Xinjiang, Inner Mongolia, Tibet et 
al have the characteristics of the sparsely populated, which can lead a poor equity in geographical dimension, this and Luo Ning, Chen Yuan consistent findings $[12,13]$.

The fairness difference mainly comes from the intra-group According to the analysis of Theil index contribution rate in the two dimensions, the intra-group contribution rate was all over $60 \%$, higher than the inter-group, which proved that the unfair difference in distribution mainly comes from within regions, which is consistent with the research results of Chang Xiang and Sun et al[14, 15].

\section{Suggestions}

Strengthen government functions in the allocation of health resources to further promote fairness

Low equity and low efficiency of health resource allocation can affect the health equity of residents[16]. To promote the equity of health resources, we need to rely not only on the market, but also on the overall arrangement of the government. Therefore, the government should strengthen its functions in the allocation of health resources, to improve the equity in the allocation of health resources in the underdeveloped areas in western China, and further realize the goal of "health equity for all" which emphasized in the "13th Five-Year Plan" and the "Outline of 
healthy China 2030 Plan".

\section{Strengthen the distribution equity in health institutions}

Health institutions play a significant role in ensuring the health of the population. Hence, a high equity of health institutions allocation is quite necessary which require the government to pay more attention to the fairness of the distribution of health institutions and improve the service capacity of health institutions to lay a solid foundation for the improvement of residents' health.

\section{Increase access to health resources by using a diversity of tools}

The equity of geographical distribution of health resources will affect the accessibility of health resources, thus affecting the health equity of residents[17]. Since the distribution of health resources in geographical dimension was not that satisfied based on the analysis results above, the government needs to strengthen the equity of health resources based on geographic through tiered medical services, medical treatment alliance, strengthen economic investment, Internet hospital and other diversity methods to improve the accessibility of health resources[18-20].

\section{Conclusion}

Base on the research above, we found that the major differences in 
allocation of heath resources were existing in geographical dimension and the intra-group (the contribution rates in intra-group were all over $60 \%)$, which may impact the accessibility of health resources. Moreover, the equity of allocation of health institutions were relatively poor, which were badly in need of promotion. And these two problems are also the key factors related to the health of people. Hence, the stakeholders which include government, health institutions and so on should pay more attention in solve the problems to improve the equity of allocation of health resources.

\section{Availability of data and materials}

The data in this paper obtained from China statistical yearbook from 2015 to 2019, and the public data which released by the National Health and Family Planning Commission from 2015 to 2019

\section{Abbreviations}

NDRC: National Development and Reform Commission

\section{References:}

[1]. Furong, L., Y. Qingqing and C. Dandi, Evaluation of Structure, Equity and Utilization Efficiency of China' s Health Resource Allocation. Medicine and Society, 2020. 33(10). 
[2]. Shuguang, S. and Z. Wangfeng, The concept, framework and path of healthy China construction. Journal of SUN YAT-SEN University (SOCIAL SCIENCE EDITION), 2020. $60(1)$ : p. $\quad 168-178$.

[3]. Yang, C. and Y. Yan, A Study on health Resource Allocation Efficiency in Jiangsu Province from the perspective of health equity. Health economics Research, 2016(12): p. 18-21.

[4]. Weixin, S., Study on the Efficiency and Equity of Health Resource Allocation in primary medical and health institutions in Jilin Province. 2020, Jilin University. p. 55 .

[5]. Yan-hui, Z., H. Xiao-ning and B. 0. Tao, Study on the Equity of Health Resources Allocation in Primary Health Care Institutions in Beijing. Chinese Health Economics, 2020. 39(7): p. 46-49.

[6]. Liu, C., Analysis on Equity of General Practitioners Allocation: Based on Gini Coefficient and Theil Index. Chinese Health Economics, 2017. 36(1): p. 49-52.

[7]. Nan, Z., et al., Analyzing the equity of health resources allocation in China based on Theil Index. / Zhang Nan, et al. / / The Chi-nese Health Service Management. Chinese Health Service Management, 2014. 31(02): p. 88-91.

[8]. Peng. R., et al., Research on the current and equity of health resource in allocation in Guangxi. Health policy research in China, 2020. 13(03): p. 57-63.

[9]. Ying, M., et al., Analysis on the Equity of Human Resource Allocation for Health in Western China: Based on the Resource Homogeneity Assumption. Chinese Health Economy, 2015. 34(7): p. 31-34. 
[10]. Xia, F., et al., Research on the Equity of Community Health Resources allocation in Jilin Province. Health Economics Research, 2016(6): p. 15-18.

[11]. Lu, M., T. Ping and X. Yukun, Analysis on the allocation efficiency of medical and health resources in 12 western provinces: Based on DEA-Malmquist index analysis. Assets and Finances In Administration and Institution, 2019(8): p. 41-42. [12]. Ning, L., et al., Analysis on the Equity Change Trend of Health Resource Allocation in Yunnan Province from 1978 to 2017. Medicine and Society, 2020. 33(2): p. $26-30$.

[13]. Yuan, C., L. Wei and C. Yu, Evaluation of Health Resource Allocation Efficiency in Yunnan Based on DEA - Malmquist Model. Administration of health services in China, 2020. $37(7)$ : p. 508-513.

[14]. Xiang, C. and C. Yang, Research of Equity of Primary Health Resources Allocation in Inner Mongolia in 2016. Medicine and society, 2019. 32(3) : p. 63-67. [15]. Sun, J. and H. Luo, Evaluation on equality and efficiency of health resources allocation and health services utilization in China. International Journal for Equity in Health, 2017. 16(1).

[16]. Wenqing, J., The Impact of Imbalanced Health Resource Allocation on the Residents' Health: Based on the Urban-Rural and Re-

gional Perspective. Health resources allocation. 35(8): p. 55-57.

[17]. Le, Y. and W. Hong-man, Analysis on the Allocation Equality in Health Service Resources in Beijing. Chinese Health Economics, 2018. 37(11): p. 37-40.

[18]. Yan, Z., et al., Analysis on the Demand and Evolution of Health Services for 
Residents in the New Period. Chinese Health Economics, 2020. 39(5): p. 5-7.

[19]. Chunyan, X. and Y. Aijie, Research on Equity of Medical and Health Resources Allocation in Shandong Province. Medicine and Society, 2018. 31(10): p. 18-21.

[20]. Dianlin, F., et al., Research on the Situation and Equity of Health Resource Allocation in Guangxi in 2015-2018. China's Health Economy, 2020. 39(06): p. 50-54.

\section{Acknowledgements}

Not applicable

\section{Funding}

Health and Health Policy Research Center of Guangxi Medical

University; 2020 Project of Guangxi Young Teachers' Ability Improvement(2020KY43004)

\section{Author information}

Zheng Wang, Doctoral candidate in School of Information and Management, Guangxi Medical University, Shuangyong Road, Nanning, Guangxi, 530021, China. Stuff of Guangxi Medical College, Kunlun Road, Nanning, Guangxi,530000, China. Email:wangzhenggx@outlook.com, Tel.: $+8618878898663$

Haoyu He, stuff of Quality Management Department, the Affiliated Hospital of Stomatology, Guangxi Medical University, 10 Shuangyong 
Road, Nanning, 530021, China Tel.:+86 18577968886

Xi Liu, Doctoral candidate in School of Information and Management, Guangxi Medical University, Shuangyong Road, Nanning, Guangxi, 530021, China. Stuff of Guangxi Medical College, Kunlun Road, Nanning, Guangxi,530000, China. Email: elsie_I@qq.com

Qiming Feng. Master supervisor and Doctoral supervisor in School of Information and Management, Guangxi Medical University, Shuangyong Road, Nanning, Guangxi, 530021, China. Email: 1179308390@qq.com Bo Wei, Master supervisor and Doctoral supervisor in School of Information and Management, Guangxi Medical University, Shuangyong Road, Nanning, Guangxi, 530021, China, Email: 583400698@qq.com

\section{Corresponding author}

Correspondence to Bo Wei, email: 583400698@qq.com

\section{Contributions}

Zheng Wang, Haoyu He and Xi Liu: Prepared all the figures and table Wrote the main manuscript test, contributions were consistent.

Qiming Feng and Bo Wei: methodology designed. All authors read and approved the final manuscript.

\section{Ethics approval and consent to participate}


The authors confirm that the ethical policies of the journal, as noted on the journal's author guidelines page, have been adhered to and the appropriate ethical review committee approval has been received.

\section{Consent for publication}

Not applicable

\section{Competing interests}

The authors declare that they have no competing interests 
Figures

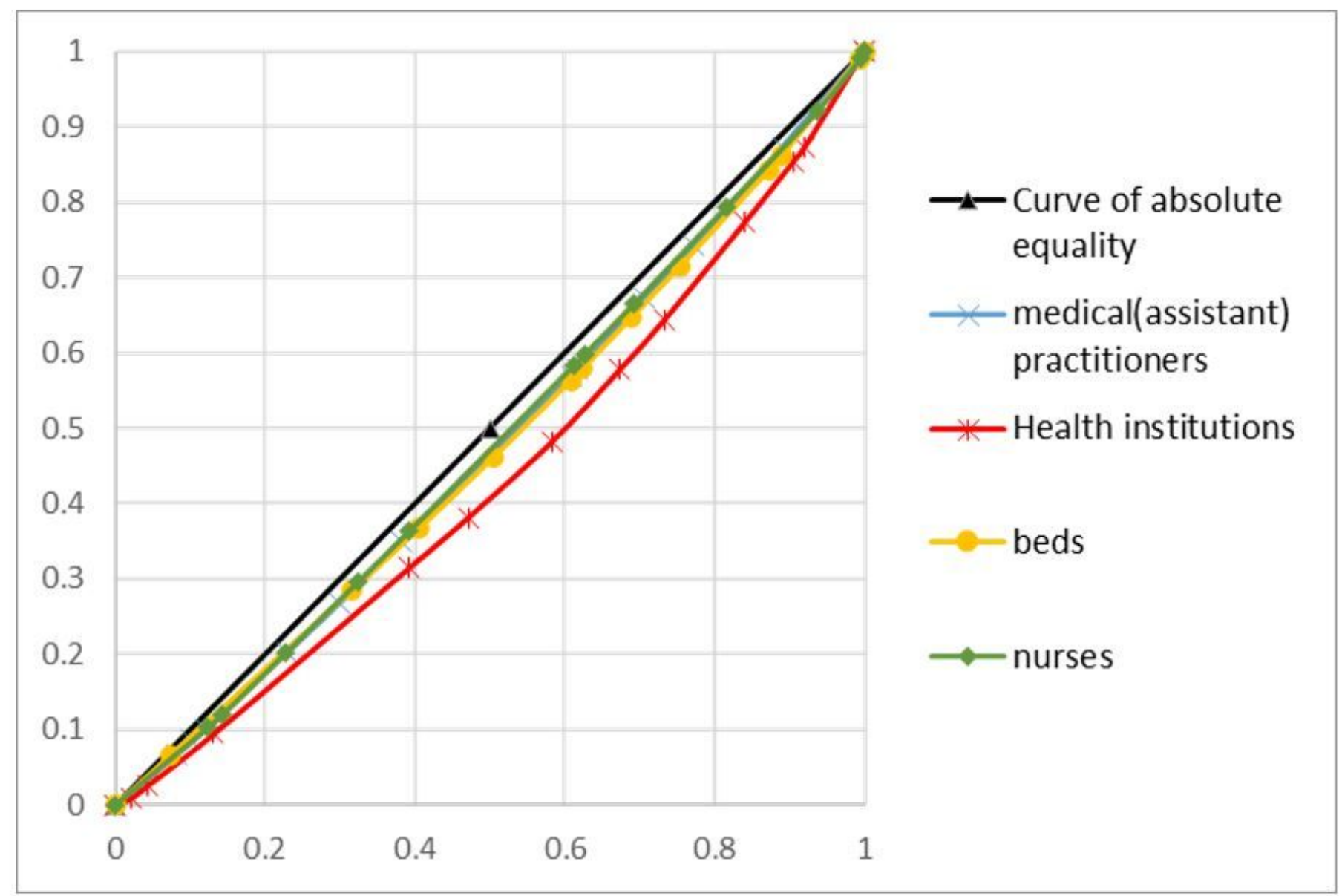

Figure 1

Lorentz curve of health resources distribution based on population dimension in western China from 2014 to 2018 


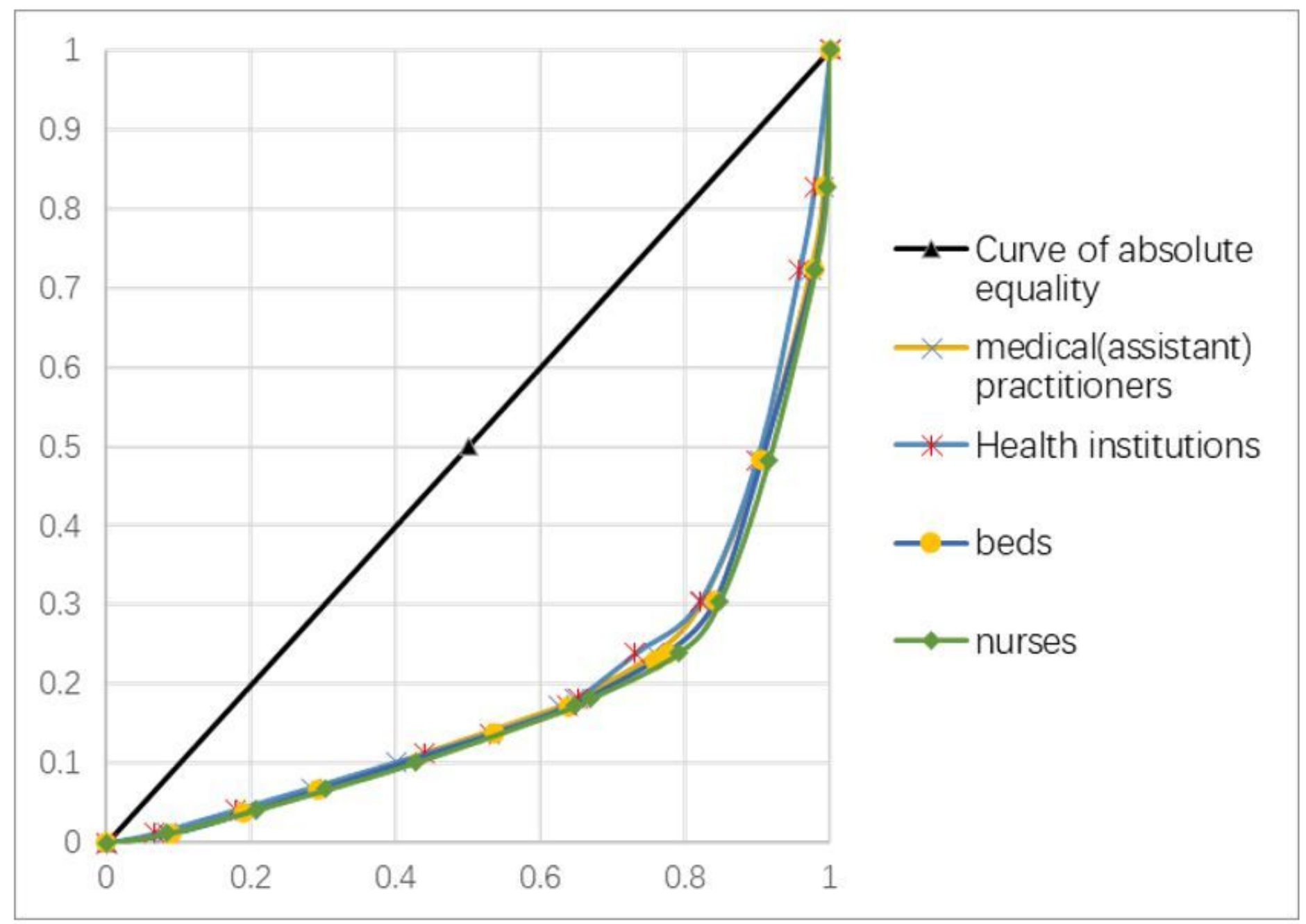

Figure 2

Lorentz curve of health resources distribution based on geographical dimension in western China from 2014 to 2018 


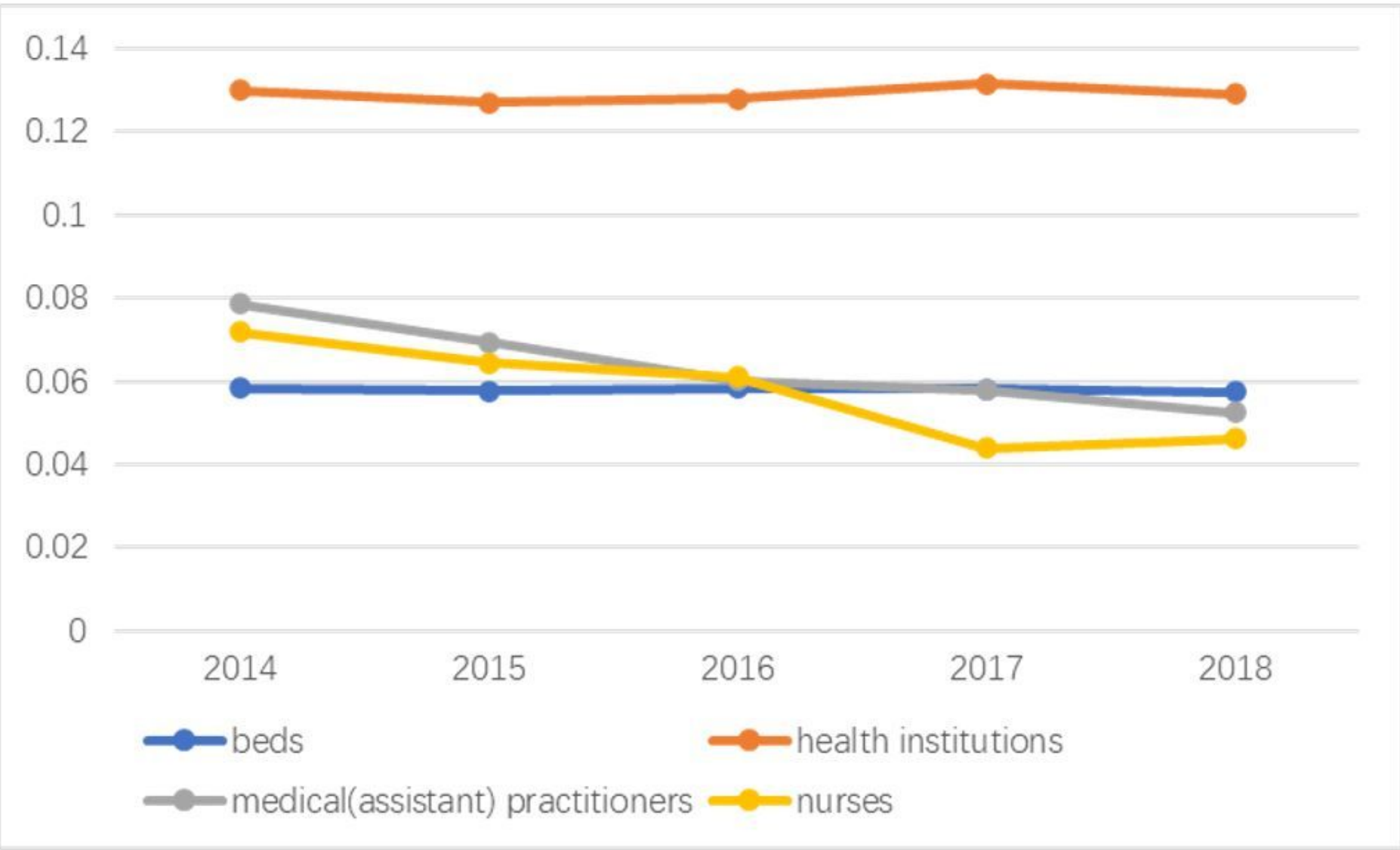

\section{Figure 3}

Gini coefficient variation in the population dimension of health resources in western China from 2014 to 2018 


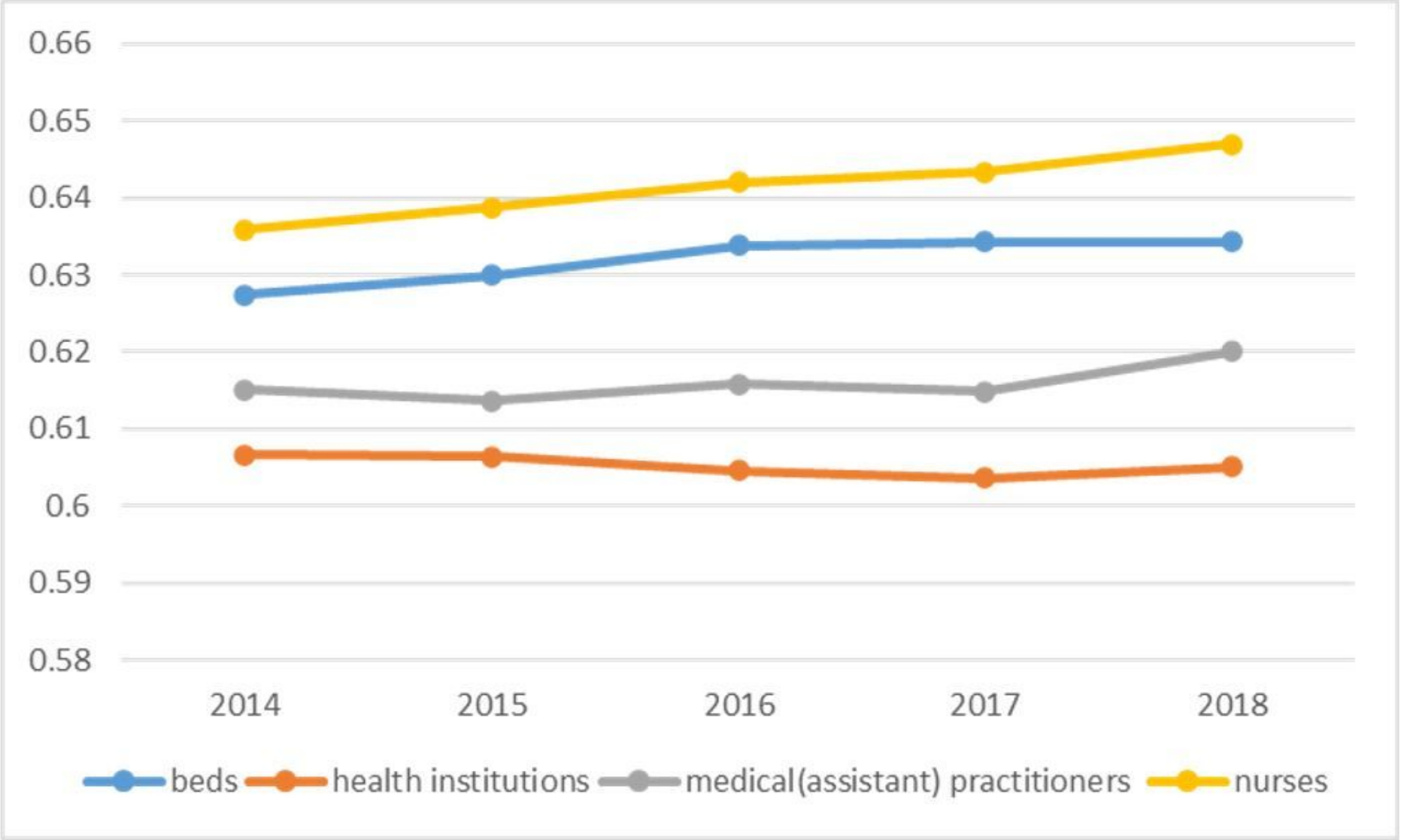

\section{Figure 4}

Gini coefficient variation in the geographical dimension of health resources in western China from 2014 to 2018

\section{Supplementary Files}

This is a list of supplementary files associated with this preprint. Click to download.

- Table1.docx

- Table2.docx

- Table3.docx

- Table4.docx

- Table5.docx

- Table6.docx

- Table7.docx

- Table8.docx 